\title{
Treatment Modalities of Odontogenic Keratocyst of Maxilla and Mandible: Our Experience
}

\author{
${ }^{1}$ Parveen Akhter Lone, ${ }^{2}$ Mohan Singh, ${ }^{3}$ Harpreet Singh Johar
}

\begin{abstract}
Background: Odontogenic keratocyst (OKC) is known for its most aggressive behavior, high recurrence rate and is most common odontogenic cyst of the jaws. After completion of odontogenesis, some remnants of dental epithelium remain in oral cavity and variety of cysts and tumor can develop from this odontogenic epithelium the most common being odontogenic keratocyst. The most common site of occurrence of OKC reported is mandibular body and ramus region.
\end{abstract}

Objectives: The aim of this study is to report the other frequent site of occurrence, different size of OKC in mandible and maxilla and different treatment modalities depending on size, age, and extent of the lesion.

Materials and methods: Ten patients were selected among the patients referred to the department of oral and maxillofacial surgery with cystic lesions on mandible and maxilla. After making diagnosis treatment planning was done depending upon the age, radiographic appearance and size of the lesion.

Results: Odontogenic keratocyst was found more in males than females, was found more in mandible than maxilla. Majority of the cases were in mandible angle ramus region followed by anterior maxilla. Seventy-six percent cases were multilocular radiolucencies, whereas $24 \%$ unilocular. Multilocular radiolucencies were treated by wide surgical excision, hemimandibulectomy, whereas unilocular radiolucencies were treated by enucleation.

Conclusion: It is concluded that treatment of OKC depends upon age, radiographic and clinical extent of lesion, unilocular or multilocular appearance, presence of daughter cysts, recurrence rate.

Keywords: Enucleation, Impacted teeth, Odontogenic epithelium, OKC, Ramus of mandible resection.

How to cite this article: Lone PA, Singh M, Johar HS. Treatment Modalities of Odontogenic Keratocyst of Maxilla and Mandible: Our Experience. World J Dent 2015;6(4):208-212.

Source of support: Nil

Conflict of interest: None

\footnotetext{
${ }^{1}$ Associate Professor and Head, ${ }^{2}$ Medical Superintendent ${ }^{3}$ House Surgeon

1,3 Department of Oral and Maxillofacial Surgery, Indira Gandhi Government Dental College, Jammu, Jammu and Kashmir, India

${ }^{2}$ Department of Hospital Administration, Indira Gandhi Government Dental College, Jammu, Jammu and Kashmir, India
}

Corresponding Author: Parveen Akhter Lone, Associate Professor and Head, Department of Oral and Maxillofacial Surgery, Indira Gandhi Government Dental College, Jammu, Jammu and Kashmir India, Phone: 09419203131, e-mail: parveen.lone@yahoo.com

\section{INTRODUCTION}

Odontogenic keratocyst (OKC) is relatively most common and most aggressive developmental odontogenic cyst of the jaw. Philipsen ${ }^{1}$ in 1956 first described this cyst and Pinborg and Hansen ${ }^{2}$ suggested the histopathological importance for the diagnosis of OKC in 1962. It was initially called as primordial cyst as tooth primordium was thought to be the origin of the lesion. It is commonly believed that the origin of OKC comes from dental lamina remnants in maxilla and mandible ${ }^{3,4}$ another origin of $\mathrm{OKC}$ is from Basel cells of oral epithelium overlying it. ${ }^{5,6}$ Odontogenic keratocyst occurs as unilocular or multilobular radiolucency often with dentigerous cyst. ${ }^{7}$ It comprises of around $11 \%$ of all cysts of jaws. The histological characteristics are very thin orthokeratinized or parakeratinized stratified squamous epithelium, well defined Basel cell layer without rete pegs, lumen containing desquamated keratin and fibrous capsule. ${ }^{4}$ In $97 \%$ of OKC parakeratinized squamous epithelial lining is seen. ${ }^{8}$ Daughter or satellite cysts are seen in connective tissue walls. Cystic lining is thin, fragile, and difficult to enucleate in one piece. In 1960, Gorlin and Goltz described simultaneous occurrence of multiple Basel cell carcinomas, multiple OKCs of mandible and maxilla bifid ribs, and other changes. ${ }^{9}$ The purpose of this study is to evaluate clinical and radiographic behavior of OKC and also various location of occurrence of the cysts.

\section{MATERIALS AND METHODS}

This present study was carried out in Department of Oral and Maxillofacial Surgery, Indira Gandhi Government Dental College, Jammu, Jammu and Kashmir, India. Ten patients were taken over period of 2 years with facial swelling, asymmetry, pain, absence of teeth in the region, trismis. Swellings on clinical examination were soft fluctuant, nontender, having smooth surface and regular borders. Various radiographs like intraoral periapical (IOPA), orthopantomography (OPG), paranasal sinus (PNS) radiographs and occlusal view was advised. For smaller unilocular cysts intraoral enucleation with primary closure, marsupialization followed enucleation was done under local anesthesia. Multilocular larger lesions were treated under general anesthesia (GA) with marginal, segmental resection and hemimandibulectomy followed by reconstruction depending upon the extent 


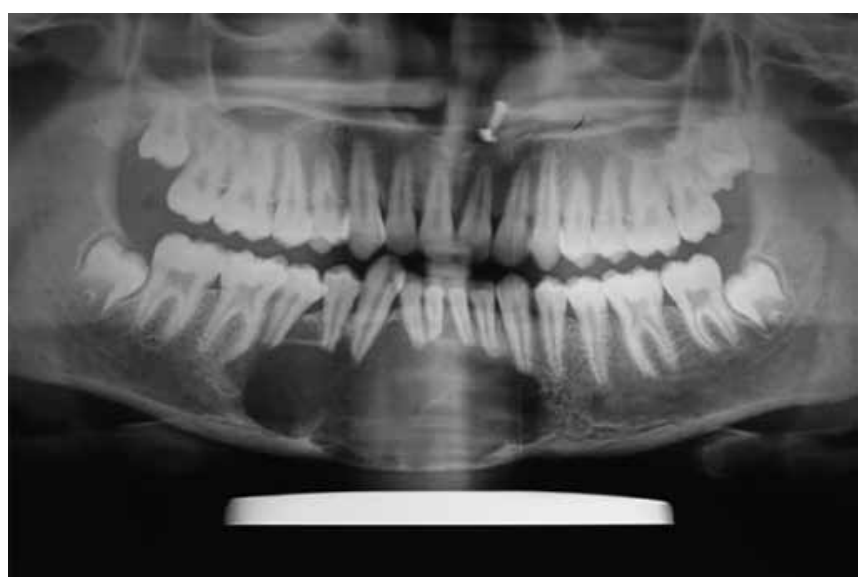

Fig. 1: Orthopantomograph showing OKC involving symphysis, parasymphysis region of mandible

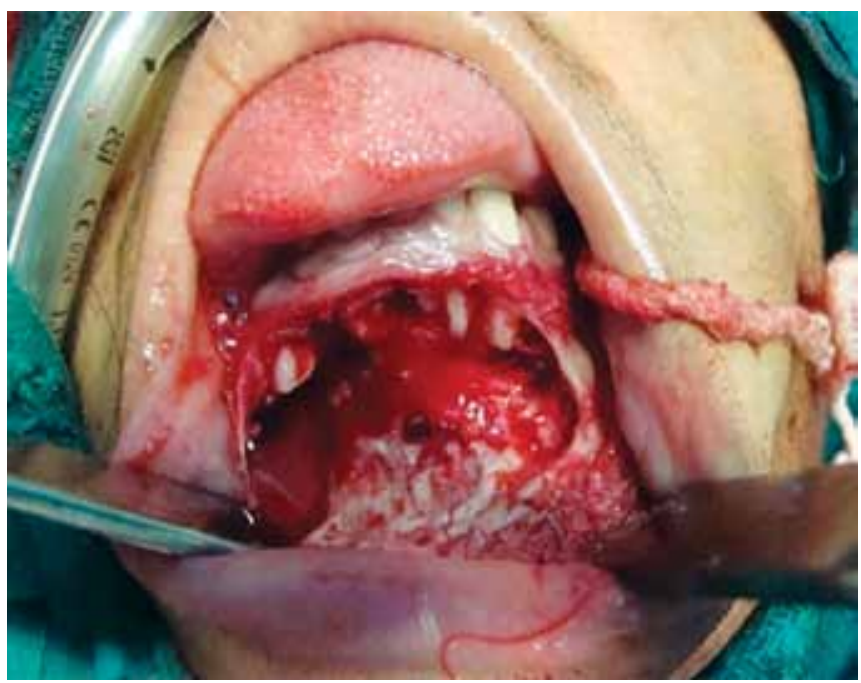

Fig. 3: Intraoperative photograph showing wide surgical excision

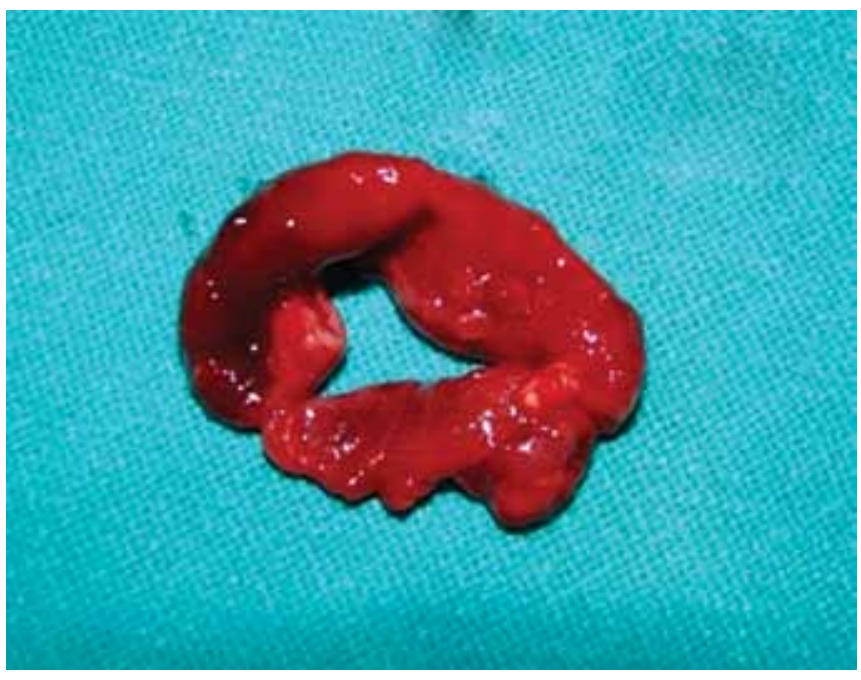

Fig. 5: Enucleation done and cystic lining sent for histopathologic examination

of lesion and indication of these procedures. Cystic lining was sent for histopathological examination which confirmed as odontogenic keratocyst (Figs 1 to 6).

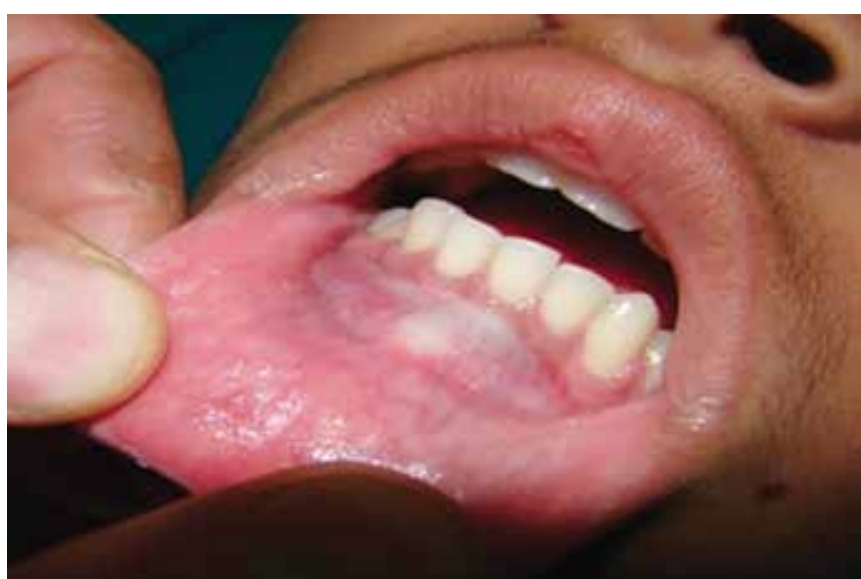

Fig. 2: Preoperative photograph showing cystic lesion in mandibular anterior region

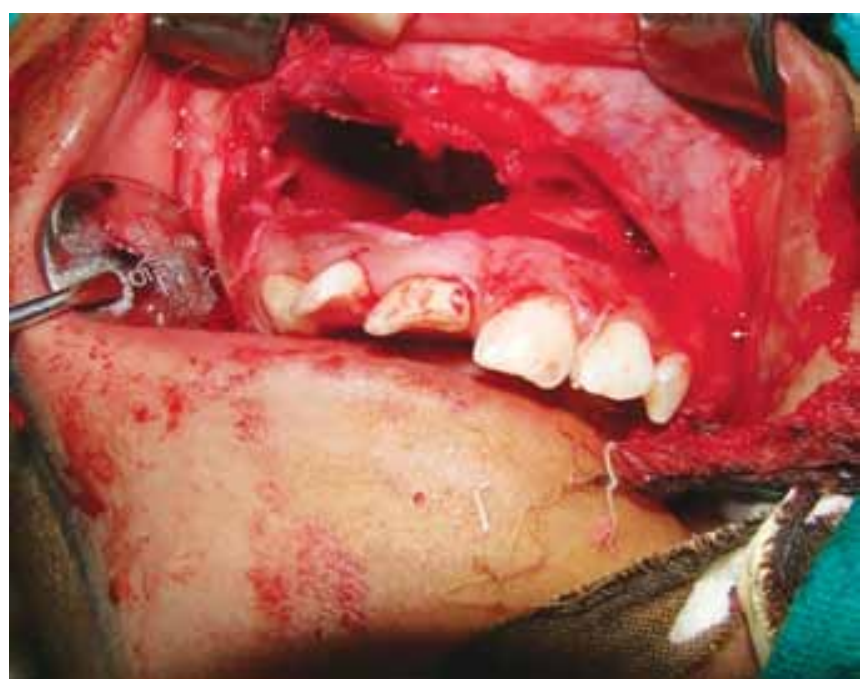

Fig. 4: Intraoperative photograph showing cystic cavity in maxilla

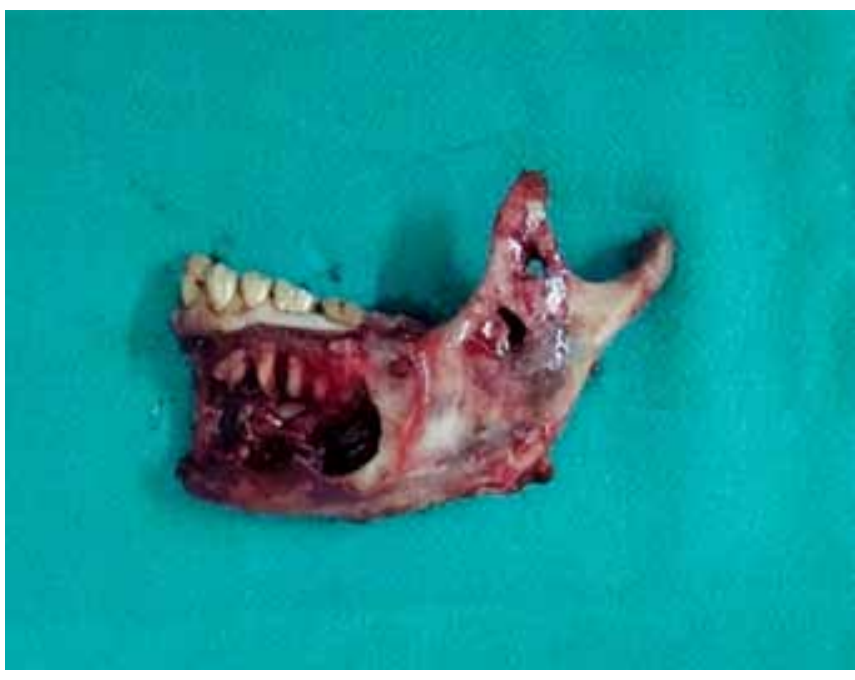

Fig. 6: Resected mandible showing OKC body of mandible involving condyle and coronoid

\section{RESULTS}

Odontogenic keratocyst was found more in males than females, male female ratio being 9:6. 
The age varied from 13 to 45 years.

The most common jaw involved in OKC was mandible.

The most common site according to the present study was the posterior mandible, i.e. the body and ramus of the mandible (7 cases), followed by the anterior mandible (4 cases).

\section{DISCUSSION}

Odontogenic keratocyst is designated by World Health Organization (WHO) as keratocystic odontogenic tumor and is defined as benign unicystic or multicystic intraosseous tumor of odontogenic origin, aggressive in nature and lined by parakeratinized stratified squamous epithelium. $^{10}$

Odontogenic keratocyst is more commonly seen in males than females. In present study, nine patients were males and six females in consistence with Renseberg et $\mathrm{al}^{11}$ similar to the present study (Fig. 7). Age range of OKC varies from first decade to 9th decade, more commonly it is seen in 2nd and 3rd decade of life ranging from 40 to $60 \%$. Many researchers have reported peak in 5 th decade of life or latter, ${ }^{12,13}$ whereas age range in the present study varies from 13 to 45 years.

Mandible is more frequently involved than maxilla. According to Shear ${ }^{14}$ out of 125 OKCs 94 was in mandible. Another study of 183 OKCs $70.5 \%$ were in mandible. Only $10 \%$ were reported in maxilla similar to the present study $70 \%$ lesions were in mandible and $30 \%$ in maxilla.

Half of the OKCs occur in mandibular angle region followed by ramus extending to body. Several studies have shown that they occur anywhere in the jaws including midline of mandible and globumaxillary region in the maxilla. ${ }^{15-17}$ Whereas in the present study out of 15 cases seven were in posterior region of mandible, four cases were in anterior mandible and one in anterior maxillary incisor region, three lesions were seen in maxillary, premolar region (Fig. 8).

Radiographically, OKC appears as unilobular or multilobular radiolucency with scalloped border. Differential diagnosis of periapical unilocular radiolucencies is other periapical or lateral periodontal cysts and dentigerous cysts. Multilocular radiolucencies should be differentiated from ameloblastoma and odontogenic myxoma. In mandible OKC tends to grow in anteroposterior direction, with displacement of unerupted teeth and displacement of mandibular canal inferiorly or superiorly can be seen. ${ }^{18}$ In the present study, $76 \%$ cysts were multilocular and $24 \%$ unilocular with well defined borders (Fig. 9).

In maxilla perforation of maxillary sinus, nasal cavity and buccal cortex can be seen ${ }^{11} 76 \%$ multilocular.

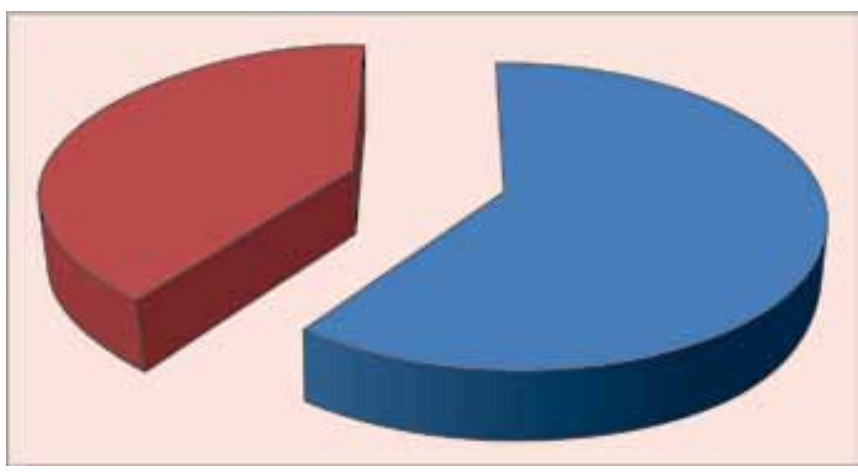

Fig. 7: Male female ratio

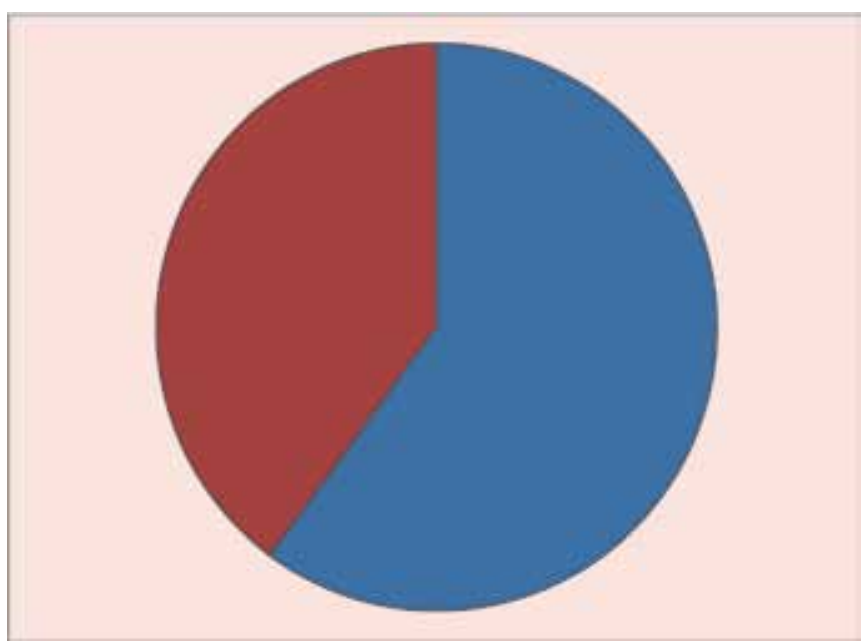

Fig. 8: One in anterior maxillary incisor and three in maxillary premolar region

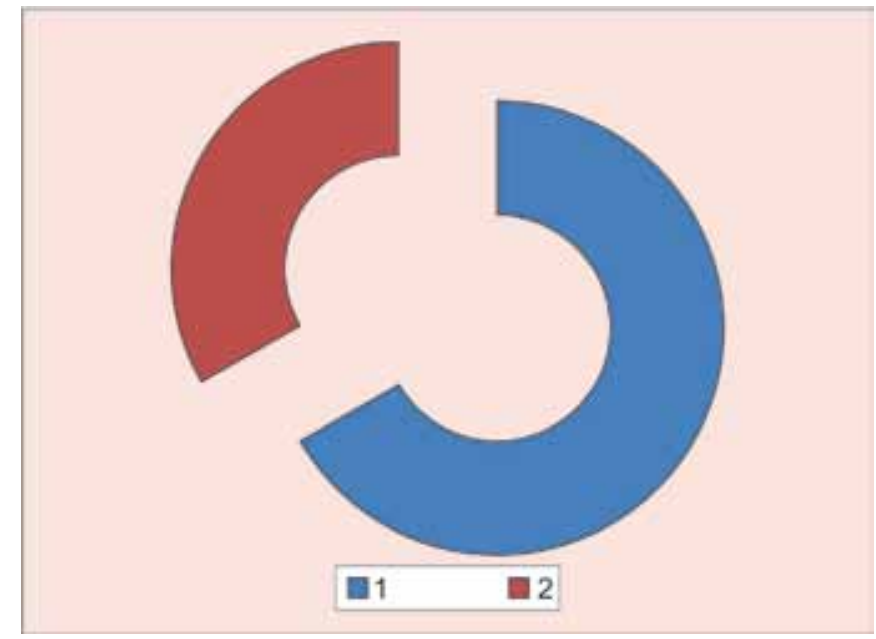

Fig. 9: Radiographically most of the cysts were multilocular $76 \%$ whereas $24 \%$ unilocular

Odontogenic keratocyst resembles dentigerous cysts, residual cysts, lateral periodontal, or periapical cysts traumatic bone cyst. Myoung et $\mathrm{al}^{19}$ reported that the radiographic diagnosis confirm histological findings in only $25 \%$ of 256 cases of OKCs.

Clinical features include swelling facial asymmetry, fluid discharge, and limited jaw opening (Graph 1). One percent limited jaw opening may be because of involvement of condylar and coronoid process. 


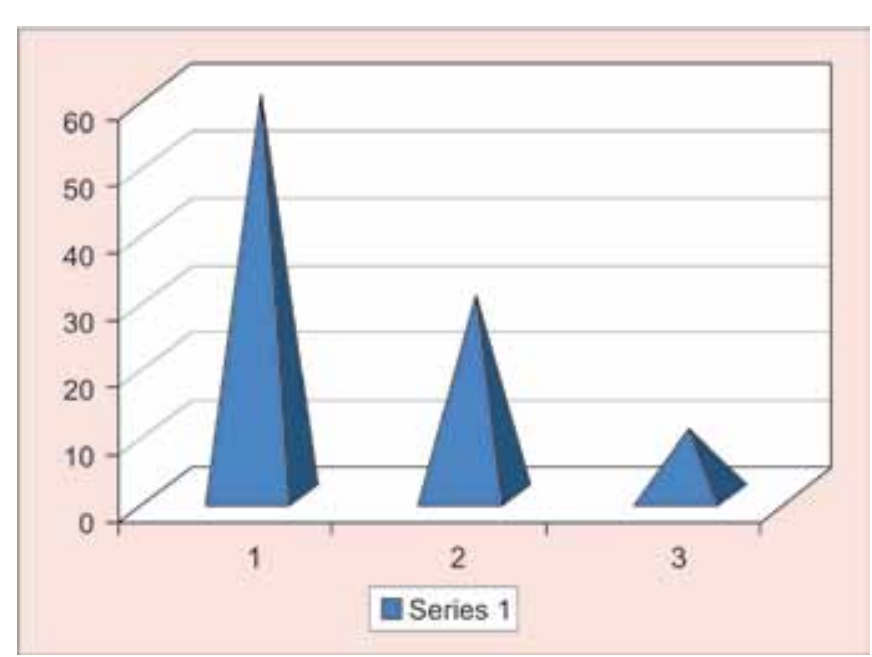

Graph 1: Most common complaint was swelling followed by fluid discharge. Restricted mouth opening as seen in one patient only

Odontogenic keratocyst occurs sporadically or in association with nevoid basal cell carcinoma syndrome (BCCS) or Gorlin syndrome. In which there are skeletal changes, basal cell carcinoma and palmer pits. ${ }^{20}$ The first clinical investigation to reveal a high propensity for recurrence was by Pinborg and Hansen in $1963^{2}$ in the present series no systemic symptoms were reported.

The treatment of OKC remains controversial. Various surgical approaches have been reported with the aim to prevent recurrence rate. Odontogenic keratocyst is known for its high recurrence rate which ranges from 5 to $62.5 \% .^{2,4,5,21}$ These include enucleation, marsupialization, decompression, curettage, followed by enucleation and treatment of defect with corny solution, liquid nitrogen cry therapy, segmental and marginal resection, and hemimandibulectomy. Meiselman et $\mathrm{al}^{21}$ favored conservative approach including enucleation, marsupilization, and curettage. The main advantage of conservative treatment is preservation of bony and associated teeth structures but the main disadvantage of decompression is its time and duration (1-14 months) during which patient looses interest in continuing treatment (proper irrigation, periodic checkup). Brannon ${ }^{4}$ stated that the recurrence rate of keratocyst, which was treated with enucleation alone, was $12 \%$. Analysis of recurrence rate suggest that it is highest with marsupialization followed by enucleation, the recurrence rate does not differ significantly between enucleation followed by primary closure and secondary granulation. ${ }^{22}$ In the present study, unilocular cysts were treated with enucleation and primary closure. No recurrence was reported.

In multilocular cysts depending upon the involvement and nature of the lesion treatment planning was done. In nonaggressive lesions curettage followed by application of corny solution to the defect was done. The cornoy solution is a tissue fixative which has ability to penetrate the bone, kills epithelial remnants and dental lamina, maintains the bony structure.

In larger and aggressive lesions involving Basel border, ramus condoyle or coronoid areas resection and hemimandibulectomy followed by reconstruction with reconstruction plate was done. Williams et $\mathrm{al}^{23}$ stressed aggressive treatment like resection with or without loss of jaw continuity resection is the only curative procedure for OKC patients with aggressive lesions and showing high recurrence rate. The concern with this method is functional and esthetic morbidity of maxilla and mandible. The present study also revealed the functional and cosmetic defects which was explained to patients in advance.

\section{CONCLUSION}

On the basis of previous studies and the present study, it is concluded that treatment of OKC depends upon age, radiographic and clinical extent of lesion, unilocular or multilocular appearance, presence of daughter cysts, recurrence rate. The aim should be to prevent recurrence, restore cosmetic and functional restoration of the patient. The recurrence rate of $\mathrm{OKC}$ is very higher than any other cysts of the jaws. Postoperative follow-up with clinical and radiological examination is necessary for at least 5 to 10 years.

\section{REFERENCES}

1. Philipsen HP. Om keratocyst (kolestetomer) I kaeberne. Tandlaegebledet 1956;60:963-981.

2. Pinborg JJ, Hansen J. Studies in odontogenic cyst epithelium: clinical and roentgen logic aspects of odontogenic keratocyst. Acts Pathol Microbiol Scand 1963;58:283-294.

3. Regezi JA, Sciubba J. Oral Pathology. Clinical-Pathologic Correlations. 2nd ed. Philadelphia, Pennsiylvania: WB Saunders Company; 1993. p. 337-341.

4. Brannon RB. The odontogenic keratocyst: a clinicopathologic study of 312 cases Part II-histological features. Oral Surg 1977;43:233.

5. Stoelinga PJ. Recurrences and multiplicity of cysts. Trans Int Conf Oral Surg 1973;4:77-80.

6. Stoelinga PJ. Studies on the dental lamina as related to its role in the etiology of cysts and tumors. J Oral Pathol 1976:65-73.

7. Sook-Bin W, Eisenbud L, Kleiman M, et al. Odontogenic keratocyst in the anterior maxilla: report of two cases, one simulating a nasopalatine cyst. Oral Surg Oral Med Oral Pathol 1987;64:463-465.

8. Manfredi M, Vescovi P, Bonanini M, Porter S. Nevoid basal cell carcinoma syndrome: a review of the literature. Int J Oral and Maxillofac Surg 2004;33:117-124.

9. Gorlin RJ, Goltz RW. Multiple nevoid basal cell epithelioma, jaw cysts, and bifid rib: a syndrome. N Engl J Med 1960; 262:908-912.

10. Barnes L, Eveson JW, Reichart P, Sidranesky D. Pathology and genetics of head and neck tumors. WHO classification of tumor series. Lyon: IARC press; 2005. p. 1-430 
11. Renseberg VL, Paquette M, Morkel AJ, Norttje JC. Correlative MRI and CT imaging of the odontogenic keratocyst: are view of twenty-one cases. Oral and Maxillofac Surg Clin N Am 2003;15:363-382.

12. Toller PA. Origin and growth of the cysts of the jaws. Ann R Coll Surg Engl 1967;40:306-336.

13. Rippin JW, Wooglar JA. The odontogenic keratocyst in BCNS and non-syndrome patients. In: Browne RM, editor. Investigative pathology of the odontogenic cysts. 1st ed. Boca Raton (FL): CRC Press; 1991. p. 221-232.

14. Shear M. Odontogenic keratocyst: clinical features. Oraland Maxillofac Surg Clin N Am 2003;15:335-345.

15. Vedtofte P, Praetorius F. Recurrence of odontogenic keratocyst of the jaws in relation to clinical and histological features: a 20 years follow-up study of 72 patients. Int J Oral Surg 1979; 8:412-420.

16. Forsell K. The primordial cyst: a clinical and radiological study. Proc Finn Dent Soc 1980;76:129-174.
17. Browne RM. The odontogenic keratocyst: histological features and their correlations with clinical behavior. Br Dent J 1971;131:249-259.

18. Stoelinga PJ. Long-term follow-up on keratocyst treated according to well defined protocol. Int J Oral Maxillofac Sugery 2001 Feb;301:14-25.

19. Myoung $\mathrm{H}$, Hong SP, Hong SD, et al. Odontogenic keratocyst: review of 256 cases for recurrence and clinicopatholgical parameters. Oral Surg Oral Med Oral Pathol Oral Radiol Endod 2001;913:328-333.

20. Shear M. The aggressive nature of the odontogenic keratocyst: is it a benign cystic neoplasm? Part 2: proliferation and genetic studies. Oral Oncol 2002;38:323-331.

21. Meiselman F. Surgical management of the odontogenic keratocyst: conservative approach. J Oral Maxillofac Surg 1994;52:960-963.

22. Voorsmit RA. The incredible keratocyst (thesis). University of Nijmegen, the Netherlands, 1984.

23. Williams TP, Connor FA. Surgical management of the odontogenic keratocyst. J Oral Maxillofac Surg 1994;52:964-967. 